\title{
Nutrition, Vitamins, Other
}

National Cancer Institute

\section{Source}

National Cancer Institute. Nutrition, Vitamins, Other. NCI Thesaurus. Code C15966.

Role of vitamins other than $A, B, C$, or $E$, their precursons, and their analogs in cancer causation or prevention and in general health. 